\title{
Supervivencia y crecimiento de Celtis tala Gillies ex Planch en la rehabilitación ecológica de canteras de conchilla abandonadas
}

\author{
Maia C. Plaza Behr ${ }^{\varpi}$; Carolina Pérez; Juan Goya \& Marcelo Arturi \\ LISEA-Universidad Nacional de La Plata, La Plata, Buenos Aires, Argentina.
}

\begin{abstract}
Resumen. En el este de la provincia de Buenos Aires, la explotación minera de los materiales calcáreos del subsuelo provoca la pérdida de cobertura de bosques nativos dominados por Celtis tala, una de las principales formaciones boscosas de la región. El objetivo de este estudio fue evaluar técnicas de plantación de tala en áreas de canteras de conchilla abandonadas, para mejorar las prácticas de recuperación de estos bosques. Como hipótesis planteamos que la supervivencia y el crecimiento son afectados de forma negativa por la competencia ejercida por especies herbáceas y por las condiciones de alta incidencia de radiación solar directa. Además, planteamos que el desmalezado, la provisión de reparo y la disponibilidad de un sustrato con mayor retención de agua favorecen tanto el crecimiento como la supervivencia. Se llevó a cabo un ensayo a campo en el que se registró la supervivencia, el diámetro a la altura de cuello y la altura de las plantas de tala a fin de evaluar la respuesta a los tratamientos de desmalezado y provisión de reparo en un gradiente de disponibilidad de sustrato fértil. La supervivencia aumentó con la profundidad del sustrato y el desmalezado aumentó el crecimiento en diámetro en los sectores con mayor profundidad de sustrato fértil y mayor biomasa de herbáceas. El reparo sólo presentó una tendencia positiva débil (no significativa) sobre el crecimiento en los sectores de mayor disponibilidad de sustrato. La pérdida de cobertura boscosa que produce la minería no se puede revertir con técnicas sencillas de plantación. Es necesario entender los procesos ecofisiológicos que limitan el establecimiento de tala, planificar el manejo del sustrato remanente y evaluar el desempeño de otras especies arbóreas locales.
\end{abstract}

[Palabras clave: talares, competencia, minería, reparo, desmalezado]

\begin{abstract}
Aвstract. Survival and growth of Celtis tala Gillies ex Planch in the ecological rehabilitation of abandoned shell quarries. In the east of the province of Buenos Aires, the mining of calcareous subsoil materials causes the loss of cover of native forests dominated by Celtis tala, one of the main forest formations in the region. The objective of this study was to evaluate the tala plantation techniques in areas of abandoned quarries, to improve the ecological rehabilitation of such areas. Our hypothesis is that survival and growth are negatively affected by the low availability of fertile soil, the competition with herbaceous plants and high the direct solar radiation incidence. We carried out a field study to estimate survival, diameter at the stem base and height of tala plants as a response to weeding and shelter in a gradient of fertile soil availability. Survival increased with soil depth, and we observed a positive response of growth rate to weeding, which was greater at high fertile soil availability. Only a weak positive effect of shelter on growth was observed at higher fertile soil availability. The loss of forest cover caused by mining cannot be reversed with simple planting techniques. It is necessary to understand the ecophysiological processes that limit the establishment of tala and to plan the management of the remaining fertile soil. Other local tree species should also be assayed.
\end{abstract}

[Keywords: talares, competition, mining, shelter, weeding]

\section{INTRODUCCIÓN}

El cambio de uso de la tierra y la explotación directa de animales, plantas y sustratos en los ecosistemas naturales son los principales impulsores de procesos de degradación y pérdida de la diversidad biológica (IPBES 2019). En particular, esto produjo una retracción de los bosques en todo el mundo, con una consecuente reducción en la provisión de los servicios ecosistémicos asociados (Haines-Young and Potschin 2010; Thompson et al. 2013). En la Argentina se identificó a la agricultura y a la ganadería como las principales presiones sobre la conservación en la mayoría de las ecorregiones del país

Editora asociada: Adriana Salvo

\mplazabehr@gmail.com
(Nanni et al. 2020) y, en las últimas décadas, la expansión de la frontera agropecuaria afectó principalmente a los bosques nativos (Montenegro 2016; García Cortés et al. 2009). La reducción de las áreas de bosque nativo provoca la disminución del almacenamiento de carbono, altera la dinámica del agua y de los nutrientes, aumenta los riesgos de erosión del suelo e implica la pérdida de biodiversidad (Ciccarese et al. 2012; Crouzeilles et al. 2016). Las estrategias adoptadas para reducir los impactos de las actividades humanas sobre los componentes naturales del paisaje tienden a evitar el uso de áreas de alto valor de conservación y a establecer acciones 
para recuperar características importantes de los sistemas ya intervenidos (Meli et al. 2017). La rehabilitación ecológica tiene como principal objetivo recomponer las funciones de un ecosistema degradado, sin que exista un planteo estricto de restablecer su estructura como meta inmediata, como sí lo pretende la restauración ecológica en sentido estricto (Aronson et al. 1993). La recuperación de la cobertura y de las funciones ecosistémicas suelen no sólo depender de la suspensión de la actividad que está generando el impacto, sino que el proceso de restauración o de rehabilitación, en muchos casos, tiene que iniciarse con intervenciones activas (Corbin and Holl 2012). Estos procesos pueden plantear grandes desafíos para la reforestación, dado que a menudo la competencia con la vegetación espontánea es importante y su manejo es altamente complejo (Stanturf et al. 2014).

Uno delos principales factores que condiciona el establecimiento de la cobertura vegetal en zonas degradadas es la disponibilidad de agua (Muñoz-Rojas et al. 2016). En la etapa de establecimiento de las leñosas, la competencia con herbáceas por este recurso se agudiza en períodos de sequía y constituye un factor crítico que puede reducir de manera notable la emergencia, el crecimiento y la supervivencia de los renovales (Scholes and Archer 1997; Riginos and Young 2007; Bhadouria et al. 2017). Los efectos negativos de la escasez de agua en ambientes degradados sobre el establecimiento de la vegetación se acentúan aun más en suelos alterados por actividades tales como la minería debido a la pérdida del sustrato fértil, el estrato con mayor capacidad de retención de agua (Bateman et al. 2016; Muñoz-Rojas et al. 2016). En los suelos muy degradados también se debe considerar el daño que provoca la alta exposición a la radiación solar. La radiación elevada puede causar la desecación de las plantas, conducirlas a temperaturas letales y producir fotoinhibición (Long et al. 1994). El desmalezado y la provisión de reparo frente a la radiación solar directa y los vientos predominantes son técnicas que pueden contribuir a mejorar las condiciones microambientales en áreas degradadas, lo que favorece la supervivencia y el crecimiento de árboles nativos (Bader et al. 2007; Riginos 2009; Sparacino et al. 2020).

En el este de la provincia de Buenos Aires, Celtis tala Gillies ex Planch (tala) forma bosques sobre cordones de conchilla (carbonato de calcio), conocidos como talares, que representan una de las principales formaciones boscosas de la provincia. Sin embargo, desde principios del siglo $X X$ han experimentado distintos procesos de degradación debido a la expansión de las fronteras urbana y agropecuaria, a la utilización de su madera como leña y a la extracción minera del material calcáreo del subsuelo sobre el que se asientan estos bosques (Parodi 1940; Torres Robles and Tur 2006). La extracción de conchilla provoca la pérdida del bosque nativo, con la consiguiente disminución de la biodiversidad y de otros servicios ecosistémicos. El cambio en el relieve que genera la explotación minera también altera las condiciones en que se desarrollan algunos procesos hidrogeoquímicos, lo cual provoca deterioro y pérdida de lentes de agua dulce que constituyen un reservorio importante del recurso hídrico en la región (Carol et al. 2015).

En ensayos de reforestación realizados en áreas de cordones desmontados con cobertura de pastizal, las plantas de tala presentaron bajo crecimiento y alta mortalidad. Estos suelos tienen poca retención de agua, lo que, combinado con falta de protección y alta competencia con el estrato herbáceo, expone a las plantas de tala a condiciones de elevada radiación solar y estrés hídrico (Azcona 2018). En claros establecidos experimentalmente en talares invadidos por Ligustrum lucidum W.T. Aiton (ligustro), sitios más protegidos respecto a los cordones desmontados con pastizal, se observó mayor supervivencia y crecimiento de los individuos plantados (Plaza Behr et al. 2016). Por otra parte, la regeneración natural de esta especie en los talares es muy baja debido a procesos de competencia con árboles adultos o con plantas herbáceas en sitios con menor cobertura arbórea (Arturi 1997). En acúmulos de tierra removida (e.g., alambrados, banquinas, cordones forestados con especies exóticas y bordes de canteras abandonadas) se observaron mayores tasas de regeneración natural. Estos sitios se caracterizan por presentar una alta disponibilidad de sustrato y baja ocupación del suelo por parte de plantas herbáceas, por lo que ofrecen mejores condiciones para el crecimiento temprano de las plántulas de tala (Arturi and Goya 2004). Sin embargo, dado que la biomasa de herbáceas aumenta con la profundidad de los suelos, las ventajas que ofrece la mayor disponibilidad de sustrato para el establecimiento de tala podrían verse contrarrestadas por el efecto competitivo que 
ejercen las plantas herbáceas. Por este motivo, los acúmulos de tierra removida representan una oportunidad para el establecimiento en el período de tiempo cercano al disturbio que lo originó (Arturi 1997). Estos resultados hacen necesario continuar con la búsqueda de alternativas de plantación y producción de plantas de tala que permitan recuperar parcialmente las funciones ecológicas del sistema anterior a la intervención minera (Arturi et al. 2012). El objetivo general de este trabajo fue evaluar técnicas de plantación de tala en una cantera de conchilla abandonada. El trabajo se desarrolló bajo la siguiente hipótesis general: la disponibilidad de agua y sustrato fértil son limitantes para el establecimiento de C. tala en canteras abandonadas por lo que la supervivencia y el crecimiento aumentarán con la provisión de reparo, el control de plantas herbáceas y la mayor disponibilidad de sustrato fértil. Se plantean como objetivos particulares evaluar a) la incidencia de la aplicación de reparo frente a la radiación solar y vientos predominantes y desmalezado frente a la competencia con herbáceas y b) el efecto de la profundidad de un sustrato con mayor contenido de materia orgánica sobre la supervivencia y el crecimiento de ejemplares de tala plantados a campo.

\section{Materiales y Métodos}

\section{Área de estudio}

Los bosques de tala se extienden sobre cordonesdeconchillaformados poringresiones marinas del Cuaternario (Cavallotto 2009), los cuales se orientan de manera paralela a la costa del Río de la Plata y forman parches de extensión variable en una matriz de zonas bajas donde se desarrollan pastizales y pajonales
(Goya et al. 1992; Arturi and Goya 2004). Debido a su biodiversidad fueron incluidos en el programa El Hombre y la Biosfera (MABUNESCO) con la creación del Parque Costero del Sur entre las localidades de Magdalena y Punta Indio (Arturi and Goya 2004). Las principales actividades económicas de la zona son la ganadería extensiva y la explotación minera de la conchilla subsuperficial. Después de la extracción de conchilla quedan alteradas las condiciones de relieve positivo y el buen drenaje del suelo, además de que se remueven los horizontes superficiales (i.e., los de mayor fertilidad y capacidad de retención de agua, en comparación con los horizontes subsuperficiales, donde predomina la conchilla). En esas condiciones se observa una reducida o nula regeneración del bosque nativo.

Este trabajo se llevó a cabo en la localidad Cerro de la Gloria, partido de Castelli (35 $\left.56^{\prime} 35.77^{\prime \prime} \mathrm{S}-57^{\circ} 26^{\prime} 48.25^{\prime \prime} \mathrm{O}\right)$ en el sector sur de la distribución de los talares de la provincia de Buenos Aires (Torres Robles and Arturi 2009) (Figura 1). La temperatura media anual es cercana a los $16^{\circ} \mathrm{C}$ y los valores anuales de precipitación son del orden de los $1000 \mathrm{~mm}$, con máximos de ocurrencia en otoño y primavera (Fucks et al. 2012). La evapotranspiración potencial es de 750 a 800 $\mathrm{mm}$ anuales, y existe una alternancia entre períodos húmedos en los que se producen inundaciones y períodos de fuertes sequías (Arturi and Goya 2004).

\section{Condiciones del sustrato}

La cantera donde se realizó el ensayo quedó inactiva a fines del año 2011 y tiene una superficie aproximada de 4 ha. Luego del

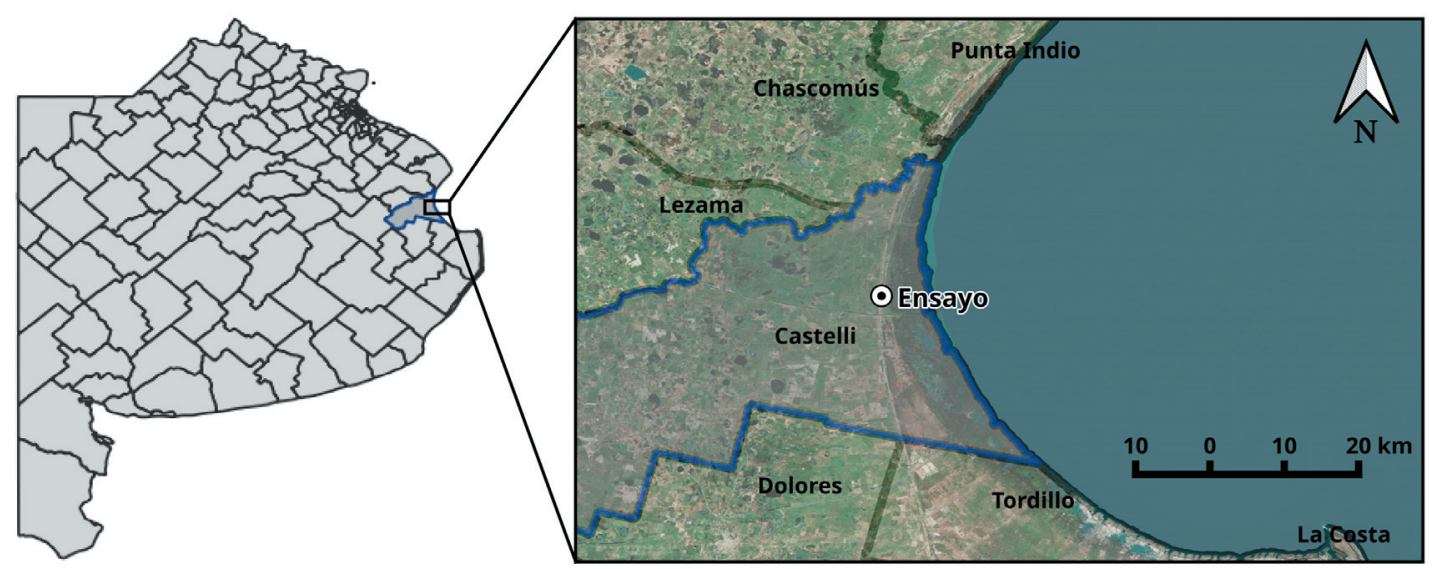

Figura 1. Ubicación del sitio de ensayo.

Figure 1. Study site location 
cese de actividades, un sector (0.5 ha) fue acondicionado con máquina retroexcavadora oruga y cargador frontal, y se dispuso sobre la superficie una capa de material limoso rico en materia orgánica (sustrato fértil) procedente de los horizontes superiores del suelo del cordón que habían sido removidos durante aproximadamente los dos años que duró la explotación minera. Este movimiento de sustratos resultó en un gradiente SE-NO de disminución de la profundidad de la capa superficial de sustrato fértil en ese sector y una fuerte variación en la textura (arenosa, franco-arenosa y arcillosa) (Figura 2). Luego de tres años el área de estudio se encontraba completamente colonizada por especies herbáceas.

\section{Ensayo de reparo y desmalezado}

En septiembre de 2014 se plantaron 48 individuos de tala de dos años de edad, producidos en vivero. Las plantas fueron obtenidas a partir de semillas colectadas en la zona de estudio y presentaban dimensiones variables entre 3 y $11 \mathrm{~mm}$ de diámetro a la altura del cuello (DAC) y alturas entre 12 y 54 $\mathrm{cm}$. Se establecieron 12 bloques con 4 plantas en un arreglo cuadrangular de $1.5 \times 1.5 \mathrm{~m}$. Cada planta recibió un tratamiento diferente resultante de la combinación de dos factores (desmalezado y reparo) con dos niveles cada uno (con tratamiento y sin tratamiento) (Figura 2). El reparo se realizó con dos tablas de $15 \times 50$ $\mathrm{cm}$ colocadas de manera vertical y orientadas hacia el noroeste con el objetivo de reducir la incidencia solar lateral directa en las horas de mayor temperatura y brindar protección contra los vientos predominantes. El desmalezado consistió en remover con pala las plantas herbáceas en un radio de $50 \mathrm{~cm}$ alrededor de la planta. Dicha remoción consistió en eliminar sólo la parte aérea de las plantas herbáceas para evitar alterar la estructura del suelo. La combinación de los dos factores determinó cuatro tratamientos: reparo, desmalezado, reparo+desmalezado y testigo sin reparo ni desmalezado. La ubicación de los tratamientos en cada bloque fue sistemática (Hurlbert 1984), pero la asignación de las plantas de tala a cada bloque y tratamiento fue aleatoria.

Durante el primer año luego de la plantación se llevaron a cabo cinco mediciones (octubre y diciembre de 2014, febrero, abril y octubre de 2015). En cada muestreo se constató la supervivencia de las plantas instaladas en el área. En las plantas vivas se midieron las variables 1) diámetro a la altura del cuello (DAC; se midió con un calibre con vernier dispuesto de forma perpendicular al eje del tallo y en la base de éste, donde comienza la parte aérea de la planta), y 2) altura total (se midió con cinta métrica la distancia perpendicular desde el plano de la base hasta la última hoja viva). Luego del muestreo de octubre de 2015 se realizaron observaciones anuales de la supervivencia de las plantas hasta diciembre de 2018.

La profundidad real del sustrato orgánico se obtuvo al finalizar el ensayo mediante 12 perforaciones en los centros de cada bloque, y se midieron con cinta métrica el límite entre la capa de sustrato fértil agregada y el material calcáreo subyacente $(n=12)$. Debido a la baja
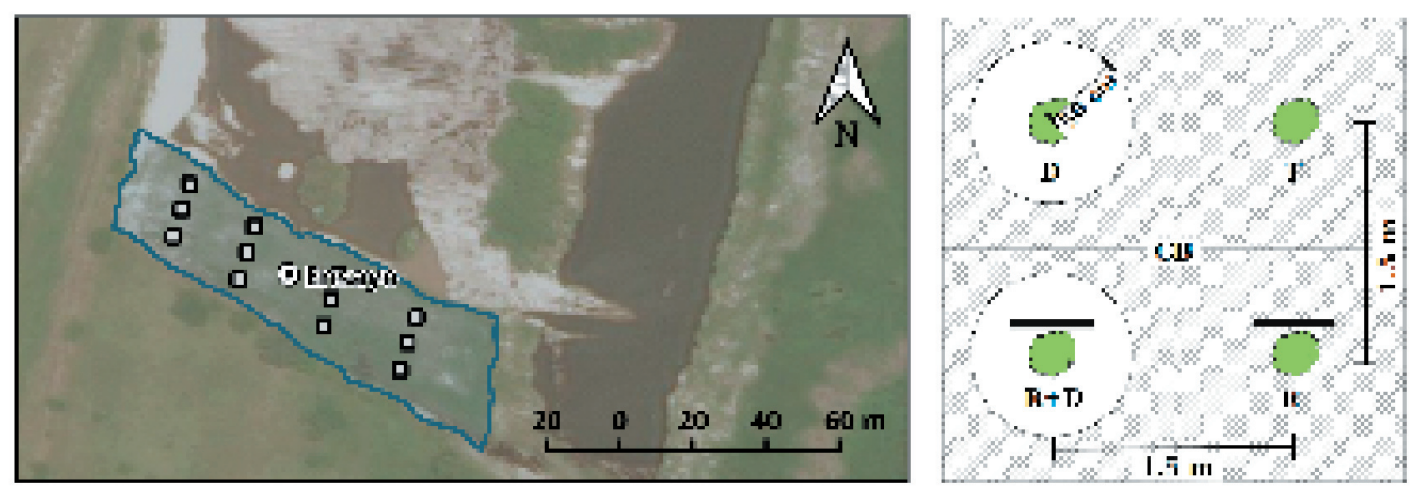

Figura 2. Distribución de los bloques del ensayo en el sitio de estudio. Los cuadrados grises indican la posición de cada bloque de cuatro individuos. A la derecha, la referencia de la posición de cada planta dentro del bloque y su tratamiento correspondiente (R: reparo; D: desmalezado; R+D: reparo y desmalezado; T: testigo). CB indica el centro del bloque.

Figure 2. Distribution of the experimental blocks at the study site. The gray squares indicate the position of each block of four plants. The position of plants and the treatment are indicated (R: shelter; D: weeding; R+D: shelter and weeding; $\mathrm{T}$ : control). CB indicates the center of the block. 
variabilidad de esa profundidad entre las parcelas de un mismo bloque se utilizó la misma estimación para todas ellas.

\section{Biomasa de herbáceas}

A fines de enero de 2015 se tomaron muestras de biomasa herbácea aérea y subterránea en cada parcela de tratamiento $(n=48)$. Para estimar la biomasa aérea se tomó una muestra circular de $54 \mathrm{~cm}^{2}$ adyacente a cada parcela de plantación. La biomasa subterránea se estimó hasta $10 \mathrm{~cm}$ de profundidad mediante un barreno para raíces (de $8.3 \mathrm{~cm}$ de diámetro y $13 \mathrm{~cm}$ de alto) colocado de manera concéntrica a la parcela de biomasa aérea. En el laboratorio se separó el material vegetal en aéreo y subterráneo, y las raíces se lavaron con agua corriente sobre tamiz para evitar la posibilidad de perder material. Las partes aérea y subterránea de cada muestra se dispusieron en bolsas de papel rotuladas, se las llevó a estufa a $60^{\circ} \mathrm{C}$ hasta peso constante y el peso seco se obtuvo con balanza de precisión.

\section{Análisis de datos}

Los datos de supervivencia se analizaron a partir del registro que detectó mortalidad de las plantas llevadas a campo. El análisis se realizó mediante una regresión logística ajustada con un modelo lineal generalizado (Faraway 2006). La variable dependiente con distribución binomial tomó los valores 1 y 0 para las plantas vivas y muertas, respectivamente, en cada fecha considerada. Las variables independientes fueron profundidad del sustrato, reparo y desmalezado. Se evaluaron las interacciones de segundo orden reparo $x$ profundidad del sustrato y desmalezado $x$ profundidad del sustrato, así como la interacción de tercer orden. La profundidad del sustrato se incluyó como variable continua y se tomó el mismo valor para todas las parcelas del mismo bloque, tanto para los análisis de supervivencia como para los de crecimiento. Estos modelos fueron ajustados mediante la función glm en el programa $\mathrm{R}$, de uso libre (R Core Team 2016) utilizando la función de enlace logit.

Se utilizaron modelos lineales mixtos para evaluar el efecto de los tratamientos de reparo y desmalezado, así como su interacción con la profundidad del sustrato, sobre la variación del DAC y la altura, que fueron calculadas como la diferencia entre las mediciones consecutivas. Dado que se analizó la diferencia entre fechas no se incluyó al tiempo como factor. Se incluyó el bloque como efecto aleatorio y se llevó a cabo un proceso de selección del modelo más apropiado según lo recomendado por West et al. (2014). Como efectos fijos se evaluaron las interacciones entre el desmalezado y la profundidad del sustrato fértil, y el reparo y la profundidad del sustrato fértil, con la finalidad de evaluar si la respuesta a los tratamientos de desmalezado y reparo difirió dependiendo de la profundidad del sustrato. Asimismo, se evaluó la interacción entre esos tres factores.

También se evaluó si la biomasa herbácea total (aérea+subterránea) aumentaba con la profundidad del sustrato mediante una regresión lineal simple. Ambas variables fueron transformadas a logaritmo natural para aproximar a una relación lineal. Para los modelos lineales mixtos se utilizó el paquete nlme (Pinheiro et al. 2015), utilizando también el entorno R. Mediante este análisis se determinó si el aumento en la disponibilidad de sustrato fértil producía una variación de la competencia con las herbáceas a fin de interpretar correctamente una eventual interacción desmalezado $\mathrm{x}$ profundidad del sustrato.

\section{Resultados}

\section{Ensayo de reparo y desmalezado}

La supervivencia de las plantas de tala fue del $100 \%$ hasta abril de 2015 . En octubre de 2015 se detectaron los primeros individuos muertos, por lo que los análisis estadísticos de la supervivencia se realizaron para ese período (abril-octubre de 2015). El tratamiento que mostró mayor supervivencia fue el desmalezado (50\%), seguido por reparo+desmalezado $(42 \%)$ y reparo $(8 \%)$; en el testigo, la supervivencia fue nula (Figura 3). Esta respuesta se vio reflejada en los análisis estadísticos, que mostraron efectos positivos significativos tanto para el desmalezado ( $b=3.5, z=2.80, P=0.0051$ ) como para la profundidad del sustrato adicionado $(b=6.8, z=2.21, P=0.0267)$, que varió entre los 7 cm y $53 \mathrm{~cm}$ de espesor entre los extremos más someros y de mayor espesor, respectivamente. No se detectaron efectos significativos para las interacciones de segundo y tercer orden. Las observaciones anuales del estado de las plantas de tala registraron una supervivencia de $14 \%$ en octubre 2016 respecto del número inicial total de individuos plantados (3 con 


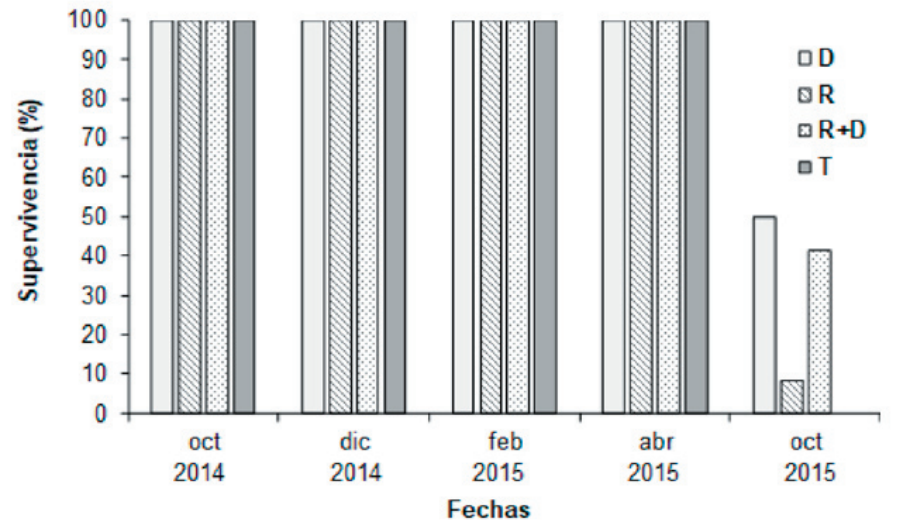

Figura 3.Supervivencia de las plantas de Celtis tala plantadas en la cantera en septiembre de 2014. R: reparo; $\mathrm{D}$ : desmalezado; $\mathrm{R}+\mathrm{D}$ : reparo y desmalezado; T: testigo. $\mathrm{N}$ inicial de cada tratamiento $=12$.

Figure 3. Survival of Celtis tala plants planted in the quarry in September 2014. R: shelter; D: weeding; R+D: shelter and weeding; $\mathrm{T}$ : control. Initial $\mathrm{N}$ of each treatment $=12$. reparo+desmalezado, 3 con desmalezado y 1 con reparo); en diciembre de 2018 sólo 6 individuos se encontraban vivos (2 con reparo+desmalezado, 3 con desmalezado y 1 con reparo). En ambas fechas, estas plantas correspondían a los bloques ubicados en el extremo de mayor profundidad del sustrato. Debido al escaso número de bloques e individuos no se realizaron análisis estadísticos luego de octubre de 2015.

El efecto de los tratamientos sobre el crecimiento de las plantas se analizó para el mismo período que para la supervivencia. El incremento en DAC fue mayor en las parcelas que recibieron tratamiento de desmalezado que en las demás parcelas, y esa diferencia fue mayor cuanto mayor fue la profundidad del sustrato, habiéndose detectado un efecto significativo de la interacción entre esos factores $(b=0.53, t=4.54, P=0.0001)$. Por otra parte, no se registró ningún efecto del reparo

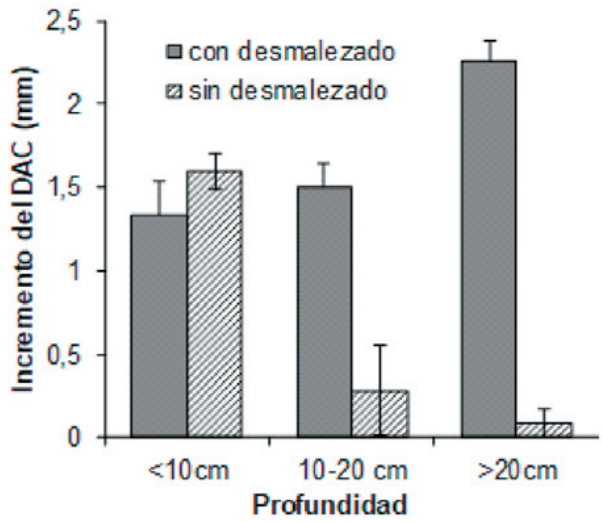

ni de su interacción con la profundidad del sustrato ni de la interacción entre los tres factores. Sin embargo, la mayor diferencia entre plantas con reparo y sin reparo en el incremento en el DAC se observó en las mayores profundidades de sustrato (Figura 4). No se retuvo el efecto aleatorio bloque en el modelo por haberse observado un menor AIC (AIC=180.4) que en el modelo ajustado con ese efecto aleatorio (AIC=184.7).

No hubo diferencias significativas entre tratamientos con respecto a la altura. Esta variable mostró una tendencia decreciente en todos los tratamientos (Figura 5). En el campo se observaban ramas secas en las porciones apicales de las plantas.

\section{Biomasa de herbáceas}

La biomasa total de herbáceas aumentó con la profundidad del sustrato $\left(R^{2}=0.23, P<0.01\right)$,

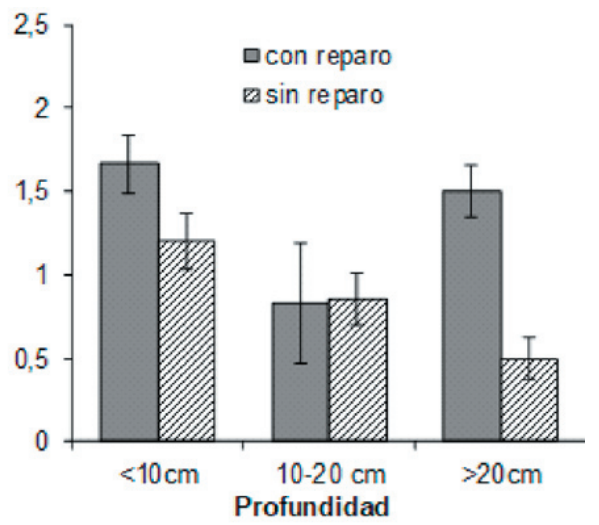

Figura 4. Incremento del DAC de Celtis tala plantados en la cantera entre octubre de 2014 y octubre de 2015 en función de la profundidad de la capa de sustrato orgánico. Las líneas verticales indican el error estándar. La profundidad del sustrato fue categorizada para presentar los resultados de los otros tratamientos en diferentes condiciones de disponibilidad de sustrato fértil.

Figure 4. Increase in DAC of Celtis tala planted in the quarry between October 2014 and October 2015 as a function of organic soil depth. The vertical lines indicate the standard error. The organic soil depth was categorized to show the effect of the other treatments in different conditions of soil availability. 

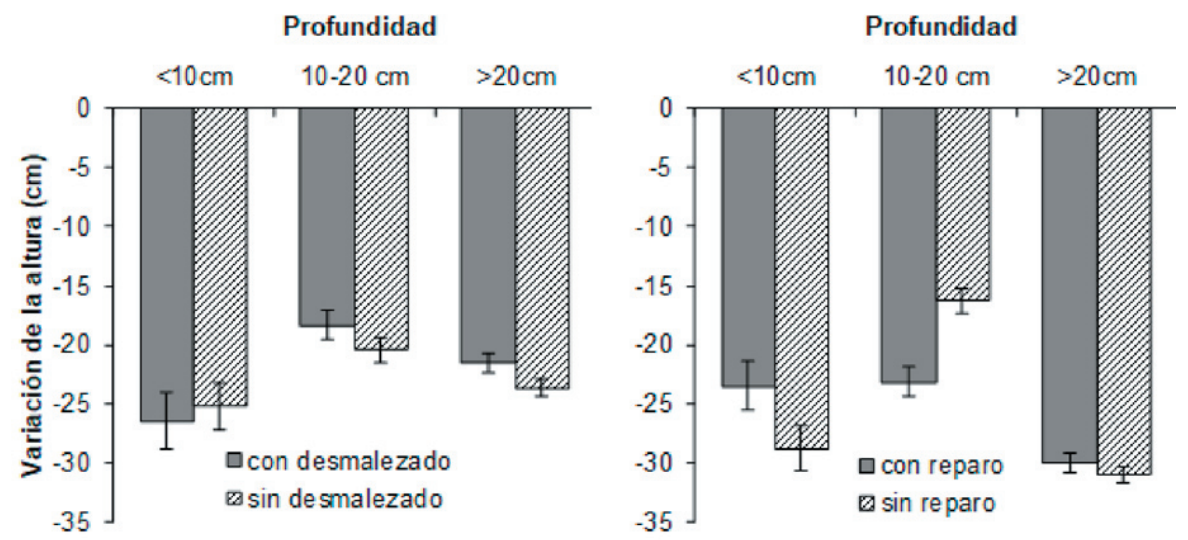

Figura 5. Variación de la altura de plantas de Celtis tala entre octubre de 2014 y octubre de 2015 en función de la profundidad de la capa de sustrato orgánico. Las líneas verticales indican el error estándar. La profundidad del sustrato fue categorizada para presentar los resultados de los otros tratamientos en diferentes condiciones de disponibilidad de sustrato fértil.

Figure 5. Height variation of Celtis tala plants between October 2014 and October 2015 as a function of organic soil depth. Vertical lines indicate standard error. The organic soil depth was categorized to show the effect of the other treatments in different conditions of soil availability.

variando entre 1 y $8 \mathrm{Mg} / \mathrm{ha}$ para los sitios con menos de $4 \mathrm{~cm}$ de sustrato fértil y entre $4 \mathrm{y}$ $>10 \mathrm{Mg} /$ ha para los sitios con más de $15 \mathrm{~cm}$ de sustrato fértil. La biomasa de raíces representó 87 a $90 \%$ de la biomasa total de herbáceas.

\section{Discusión}

La competencia con herbáceas y la variación en la disponibilidad de sustrato afectaron a la supervivencia y el crecimiento de las plantas de tala. El efecto de la competencia que producen las herbáceas pudo detectarse por el efecto positivo del desmalezado sobre la supervivencia y el crecimiento. En términos de supervivencia, los porcentajes de plantas vivas en los tratamientos con desmalezado fueron significativamente mayores que en los restantes. Estos resultados son coherentes con las tendencias de la regeneración natural de la especie, ya que el establecimiento espontáneo de tala es más frecuente en sitios donde la biomasa total de raíces es baja o nula, comparada con el bosque nativo y los pastizales (Arturi and Goya 2004). En sabanas de Kenia, Riginos (2009) evaluó, mediante experimentos de remoción, el efecto de los pastos sobre el crecimiento de árboles de $30 \mathrm{~cm}$ a más de $4 \mathrm{~m}$ de altura, y encontró que la competencia de los pastos redujo de forma marcada el crecimiento de los árboles, incluso en aquellos pertenecientes a las clases de tamaño mayores. Las zonas de enraizamiento de ambos tipos biológicos mostraban una importante superposición en la ocupación del suelo (Riginos 2009). Las plantas que no recibieron desmalezado tendieron a presentar menor crecimiento en los sitios con mayor profundidad de sustrato, que a su vez presentaron mayor biomasa de herbáceas, y las que sí recibieron desmalezado tendieron a aumentar su crecimiento en ese mismo gradiente. Esas tendencias sugieren que el efecto negativo de la competencia con herbáceas sobre el crecimiento tendió a ser mayor que el efecto positivo del incremento en la disponibilidad de sustrato fértil.

El reparo no demostró tener un efecto positivo sobre la supervivencia y el crecimiento. Esto podría relacionarse con que si bien se restringió la incidencia de la radiación directa en las horas de mayor temperatura, es probable que sea necesaria una protección mayor contra la radiación solar y el viento. El tratamiento de reparo tampoco presentó una interacción significativa con el desmalezado ni con la profundidad del sustrato fértil. Sin embargo, de manera similar a lo observado respecto del tratamiento de desmalezado, las plantas que no recibieron tratamiento de reparo tendieron a presentar menores crecimientos en sitios con mayor profundidad de sustrato fértil, los que, a su vez, presentaron mayor biomasa de herbáceas. En los sitios ubicados en el extremo de ese gradiente de profundidad de sustrato fértil es donde se observó la mayor diferencia de crecimiento en DAC en favor de las plantas que recibieron tratamiento de reparo. Esa tendencia no fue significativa pero sugiere que el reparo podría haber representado una mejora de las condiciones de crecimiento en la situación de mayor competencia, sin embargo se requiere explorar diferentes mecanismos 
para proveer reparo más eficientemente. Existen otros tipos de protección, pero cabe mencionar que el material utilizado como reparo en actividades de rehabilitación además de ser de bajo costo debería poder permanecer en los sitios intervenidos (biodegradable). Por otra parte, el efecto de dos tipos de reparo fue evaluado en ambientes semi-áridos por Oliet et al. (2019). Estos autores señalan que el efecto del tipo de reparo depende de la especie. Por ello, en futuros estudios, además de ponerse a prueba medios de reparo que otorguen una mayor protección, deberían evaluarse otros materiales y la sensibilidad del efecto del reparo con sensores de humedad y temperatura. Arturi y Goya (2004) observaron regeneración natural de tala en plantaciones de Eucalyptus spp. La biomasa de herbáceas y raíces de arbóreas en esas situaciones fue más de 10 veces inferior a la observada en el bosque nativo (Arturi 1997), por lo que el éxito de la regeneración en esas plantaciones podría deberse a la baja competencia y al reparo provisto por una estructura forestal madura. Además, en claros generados en bosques de tala invadidos por ligustro, ensayos de reforestación con tala evidenciaron un efecto positivo de la protección del bosque aledaño, comparado con la plantación realizada en cordones desmontados (Plaza Behr et al. 2016; Azcona 2018).

En este ensayo, luego de un año desde la plantación, la supervivencia fue menor a $25 \%$, lo que indica que los factores que provocan mortalidad no fueron controlados en la medida necesaria, o bien que existen otros factores que afectan a la supervivencia de tala. La incidencia de la profundidad del sustrato fértil fue marcada, ya que $\sim 70 \%$ de las plantas que sobrevivieron hasta octubre de 2015 se encontraban en posiciones del terreno con mayor espesor de sustrato fértil. En diciembre de 2018, las plantas ubicadas en esta parte del terreno representaban el $100 \%$ de las sobrevivientes. Las plantas de tala evidenciaron una disminución en altura independientemente del tratamiento y de la profundidad del sustrato fértil. Las porciones apicales muertas observadas a campo podrían atribuirse a daños por factores climáticos (e.g., heladas, radiación solar, vientos).
En síntesis, el ensayo a campo permitió encontrar resultados parcialmentecoincidentes con la hipótesis planteada, lo cual indica que existe un efecto negativo de la competencia producida por las herbáceas y otro positivo de la disponibilidad de un sustrato de mejor calidad sobre la supervivencia y crecimiento de plantas de tala. El efecto positivo del reparo sólo manifestó una débil tendencia, lo cual sugiere que probablemente las condiciones experimentales no hayan sido apropiadas para ponerlo de manifiesto.

Estos resultados ponen en evidencia que las condiciones ambientales de los sitios explotados no resultan adecuadas para el establecimiento de tala, aun con el agregado de una capa superficial de sustrato fértil. En consecuencia, la pérdida de cobertura boscosa que produce esta actividad no puede revertirse con técnicas sencillas de plantación. Los futuros esfuerzos deben dirigirse a entender diferencias ecofisiológicas entre las plantas de tala en diferentes estados de desarrollo y a generar técnicas eficientes de reducción de la competencia, mejora del sustrato y provisión de un microclima apropiado. Enmarcado en un enfoque de rehabilitar las condiciones de cobertura arbórea anterior al disturbio, se podría sugerir analizar la plantación de otras especies de árboles de la región y utilizar plantas nodrizas que provean condiciones adecuadas para el establecimiento. Tales técnicas deberían resultar apropiadas en costo y mejora del paisaje, ya que el éxito de los proyectos de rehabilitación ecológica no sólo depende de las limitantes bióticas y abióticas propias del sistema, sino que, además, deben resultar económicamente viables y socialmente aceptables (Lamb and Gilmour 2003).

Agradecimientos. Este trabajo fue financiado por subsidios de la UNLP del Proyecto de Incentivos 11/A264, por la Facultad de Ciencias Agrarias y Forestales y por la empresa Domingo González y Cía. S.A. Agradecemos la valiosa participación en tareas de campo de estudiantes de la Facultad de Cs. Agrarias y Forestales y de la Facultad de Cs. Naturales y Museo de la Universidad Nacional de La Plata. Agradecemos a los y las revisoras anónimas $\mathrm{y}$ a la editora por sus valiosos aportes para mejorar este manuscrito. 


\section{REFERENCIAS}

Aronson, J., C. Floret, E. Floc'h, C. Ovalle, and R. Pontanier. 1993. Restoration and Rehabilitation of Degraded Ecosystems in Arid and Semi-Arid Lands. I. A View from the South. Restoration Ecology 1:8-17. https://doi.org/10.1111/j.1526 100X.1993.tb00004.X

Arturi, M. F. 1997. Regeneración de Celtis tala y su relación con el pastoreo, la cobertura herbácea y arbórea en el NE de la provincia de Buenos Aires, Argentina. Ecología Austral 7:3-12.

Arturi, M. F., and J. F. Goya. 2004. Estructura, dinámica y manejo de los talares del NE de Buenos Aires. Pp. 1-23 en Arturi, M. F., J. L. Frangi and J. F. Goya (eds.). Ecología y manejo de los bosques de Argentina. Editorial de la Universidad Nacional de La Plata, La Plata, Buenos Aires, Argentina.

Arturi, M. F., J. F. Goya, C. Pérez, S. L. Burns, M. Azcona, M. Presutti, C. Graciano, and M. Cavalcante. 2012. Estrategias para la recuperación de áreas degradadas y alternativas de manejo forestal en los talares de Magdalena y Punta Indio. Proyecto Institucional de Investigación y Extensión de la Facultad de Ciencias Agrarias y Forestales. Informe final inédito.

Azcona, M. 2018. Evaluación de técnicas de rehabilitación de áreas degradadas en los talares de Magdalena y Punta Indio. Trabajo final de grado. Facultad de Ciencias Agrarias y Forestales, Universidad Nacional de La Plata, La Plata, Buenos Aires. Pp. 39.

Bader, M. Y., I. van Geloof, and M. Rietkerk. 2007. High solar radiation hinders tree regeneration above the alpine treeline in northern Ecuador. Plant Ecology 191:33-45. http://doi.org/10.1007/s11258-006-9212-6.

Bhadouria, R., R. Singh, P. Srivastava, S. Tripathi, and A. S. Raghubanshi. 2017. Interactive effect of water and nutrient on survival and growth of tree seedlings of four dry tropical tree species under grass competition. Tropical Ecology 58:611-621.

Bateman, A., W. Lewandrowski, J. Stevens, and M. Muñoz-Rojas. 2016. The limitations of seedling growth and drought tolerance to novel soil substrates in arid systems: Implications for restoration success. Geophysical Research Abstracts 18:EGU2016-5557.

Cavallotto, J. 2009. Caracterización e historia evolutiva del sustrato sobre el que se desarrollan los talares del nordeste bonaerense. Páginas 66-81 en Athor, J. (ed.). Parque costero del sur: Magdalena y Punta Indio. Fundación de Historia Natural Félix de Azara, Buenos Aires, Argentina.

Ciccarese, L., A. Mattsson, and D. Pettenella.2012. Ecosystem services from forest restoration: thinking ahead. New Forests 43:543-560. https://doi.org/10.1007/s11056-012-9350-8.

Corbin, J. D. and K. D. Holl. 2012. Applied nucleation as a forest restoration strategy. Forest Ecology and Management 265:37-46. https://doi.org/10.1016/i.foreco.2011.10.013.

Crouzeilles, R., M. Curran, M. S. Ferreira, D. B. Lindenmayer, C. E. Grelle, and J. M.R. Benayas. 2016. A global metaanalysis on the ecological drivers of forest restoration success. Nature communications 7:11666. https://doi.org $10.1038 /$ ncomms11666.

Díaz, S., J. Settele, E. S. Brondízio, H. T. Ngo, M. Guèze, J. Agard, A. Arneth, P. Balvanera, K. A. Brauman, S. H. M. Butchart, K. M. A. Chan, L. A. Garibaldi, K. Ichii, J. Liu, S. M. Subramanian, G. F. Midgley, P. Miloslavich, Z. Molnár, D. Obura, A. Pfaff, S. Polasky, A. Purvis, J. Razzaque, B. Reyers, R. Roy Chowdhury, Y. J. Shin, I. J. Visseren-Hamakers, K. J. Willis, and C. N. Zayas (eds.). Summary for policymakers of the global assessment report on biodiversity and ecosystem services of the Intergovernmental Science-Policy Platform on Biodiversity and Ecosystem Services. IPBES 2019. IPBES secretariat, Bonn, Germany.

Faraway, J. J. 2006. Extending the linear model with r: generalized linear, mixed effects and nonparametric regression models. Chapman and Hall. Florida, EE.UU.

Fucks, E., F. Pisano, J. Carbonari, and R. Huarte. 2012. Aspectos geomorfológicos del sector medio e inferior de la Pampa Deprimida, provincia de Buenos Aires. Revista de la Sociedad Geológica de España 25:107-118.

García Cortés, M., C. Pérez, M. Presutti, and M. Arturi. 2009. Cambios en la superficie boscosa y biomasa forrajera en los talares de Magdalena y Punta Indio. Pp. 92-103 en Athor, J. (ed.). Parque Costero del Sur. Naturaleza, Conservación y Patrimonio Cultural. Fundación de Historia Natural Félix de Azara, Buenos Aires, Argentina.

Goya, J. F., G. Placci, M. F. Arturi, and A. Brown. 1992. Distribución y características estructurales de los Talares de la Reserva de Biosfera Parque Costero del Sur. Revista de la Facultad de Agronomía, La Plata 68:53-64.

Haines-Young, R., and M. Potschin. 2010. The links between biodiversity, ecosystem services and human well-being. Pp. 110-139 in Raffaelli, D. and C. Frid (eds.). Ecosystem Ecology: a new synthesis. BES Ecological Reviews Series, Cambridge University Press, UK. https://doi.org/10.1017/CBO9780511750458.007.

Hurlbert, S. H. 1984. Pseudoreplication and the design of ecological field experiments. Ecological Monographs 54: 187-211. https://doi.org/10.2307/1942661.

Lamb, D., and D. Gilmour. 2003. Rehabilitation and restoration of degraded Forests. IUCN, Gland, Switzerland and Cambridge, UK and WWF, Gland, Switzerland.

Long, S. P., S. Humphries, and P. G. Falkowski. 1994. Photoinhibition of photosynthesis in nature. Annual Review of Plant Biology 45:633-662. https://doi.org/10.1146/annurev.pp.45.060194.003221.

Meli, P., F. F. Herrera, F. Melo, S. Pinto, N. Aguirre, K. Musálem, C. Minaverry, W. Ramírez, and P. H. Brancalion. 2017. Four approaches to guide ecological restoration in Latin America. Restoration Ecology 25:156-163. https: /doi.org/10.1111/rec.12473.

Montenegro, C. 2016. Monitoreo de la Superficie de Bosque Nativo de la República Argentina. Regiones Forestales Parque Chaqueño, Yungas, Selva Paranaense y Espinal. Unidad de Manejo del Sistema de Evaluación Forestal, 
Dirección de Bosques, Secretaría de Política Ambiental, Cambio Climático y Desarrollo Sustentable. Ministerio de Ambiente y Desarrollo Sustentable de la Nación. Buenos Aires, Argentina.

Muñoz-Rojas, M., T. Erickson, D. Martini, K. Dixon, and D. Merritt. 2016. Climate and soil factors influencing seedling recruitment of plant species used for dryland restoration. Soil 2:287-298. https://doi.org/10.5194/soil-2-287-2016.

Nanni, A. S., M. Piquer-Rodríguez, D. Rodríguez, M. Nuñez-Regueiro, M. E. Periago, S. Aguiar, S. A. Ballari, C. Blundo, E. Derlindati, Y. Di Blanco, A. Eljall, R. H. Grau, L. Herrera, A. Hurtas Herrera, A. E. Izquierdo, J.Lescano, L. Macchi, F. Mazzini, M. Milkovic, L. Montti, A. Paviolo, M. Pereyra, R. D. Quintana, V. Quiroga, D. Renison, M. Santos Beade, A. Schaaf, and N. I. Gasparri. 2020. Presiones sobre la conservación asociadas al uso de la tierra en las ecorregiones terrestres de la Argentina. Ecología Austral 30:304-320. https://doi.org/10.25260/EA.20.30.2.0.1056.

Oliet, J. A., R. Blasco, P. Valenzuela, M. M. de Blas, and J. Puértolas. 2019. Should we use meshes or solid tube shelters when planting in Mediterranean semiarid environments? New Forests 50:267-282. https://doi.org/10.1007/s11056018-9659-4

Plaza Behr, M. C., C. Pérez, J. F. Goya, M. Azcona, and M. F. Arturi. 2016. Plantación de Celtis ehrenbergiana como técnica de recuperación de bosques invadidos por Ligustrum lucidum en los talares del NE de Buenos Aires. Ecología Austral 26:171-177. https://doi.org/10.25260/EA.16.26.2.0.176.

Pinheiro, J., D. Bates, S. DebRoy. D. Sarkar. and R Core Team. 2015. nlme: Linear and Nonlinear Mixed Effects Models. $\mathrm{R}$ package version 3.1-122. https://CRAN.R-project.org/package=nlme

R Core Team. 2016. R: A language and environment for statistical computing. R Foundation for Statistical Computing, Vienna, Austria.

Riginos, C. 2009. Grass competition suppresses savanna tree growth across multiple demographic stages. Ecology 90: 335-340. https://doi.org/10.1890/08-0462.1

Riginos, C., and T. P. Young. 2007. Positive and negative effects of grass, cattle, and wild herbivores on Acacia saplings in an East African savanna. Oecologia 153:985-995. https://doi.org/10.1007/s00442-007-0799-7.

Scholes, R. J., and S. R. Archer. 1997. Tree-grass interactions in savannas. Annual Review of Ecology and Systematics 28:517-544. https://doi.org/10.1146/annurev.ecolsys.28.1.517.

Sparacino, J., D. Renison, A. M. Devegili, and R. Suárez. 2020. Wind protection rather than soil water availability contributes to the restriction of high-mountain forest to ravines. New Forests 51:101-117. https://doi.org/10.1007/ s11056-019-09722-z.

Stanturf, J. A., B. J. Palik, and R. K. Dumroese. 2014. Contemporary forest restoration: a review emphasizing function. Forest Ecology and Management 331:292-323. https://doi.org/10.1016/i.foreco.2014.07.029.

Thompson, I. D., M. R. Guariguata, K. Okabe, C. Bahamondez, R. Nasi, V. Heymell, and C. Sabogal.2013.An operational framework for defining and monitoring forest degradation. Ecology and Society 18(2):20. hhttps://doi.org/10.5751/ES05443-180220.

Torres Robles, S. S., and M. F. Arturi. 2009. Variación de la composición y riqueza florística en los talares del parque costero del sur y su relación con el resto de los talares bonaerenses. Pp. 104-121 en Athor J. (ed.). Parque Costero del Sur. Naturaleza, Conservación y Patrimonio Cultural. Fundación de Historia Natural Félix de Azara, Buenos Aires, Argentina.

Torres Robles, S. S., and N. M. Tur. 2006. Los talares de la provincia de Buenos Aires. Pp. 246-250 en Brown, A., U. Martínez Ortiz, M. Acerbi and J. Corcuera (eds.). La Situación Ambiental Argentina 2005. Fundación Vida Silvestre Argentina, Buenos Aires, Argentina.

West, B., K. B. Welch, A. T. Galecki. 2014. Linear Mixed Models: A Practical Guide Using Statistical Software. Second Edition. Chapman and Hall, Boca Raton, Florida, USA. https://doi.org/10.1201/b17198. 